\title{
EXPERIMENTAL SURVEY OF THE L-H TRANSITION CONDITIONS IN THE DIII-D TOKAMAK
}

T.N. CARLSTROM, P. GOHIL, J.G. WATKINS, $(a)$ K.H. BURRELL, S. CODA,(b) E.J. DOYLE,(c) R.J. GROEBNER, J. KIM, R.A. MOYER, (c) and C.L. RETTIG(c)

This is a preprint of a paper to be presented at the Fourth H-Mode Workshop, November 15-17, 1993, Naka, Japan, and to be printed in Plasma Physics and Controlled Fusion.
Work supported by
U.S. Department of Energy
Contract Nos. DE-AC03-89ER51114, DE-FG03-89ER51121, DE-AC02-78ET51013, and DE-ACO32-76DP00789
(a) Sandia National Laboratories.
(b) Massachusetts Institute of Technology.
(c) University of California, Los Angeles.

\section{GENERAL ATOMICS PROJECT 3466 JANUARY 1994}

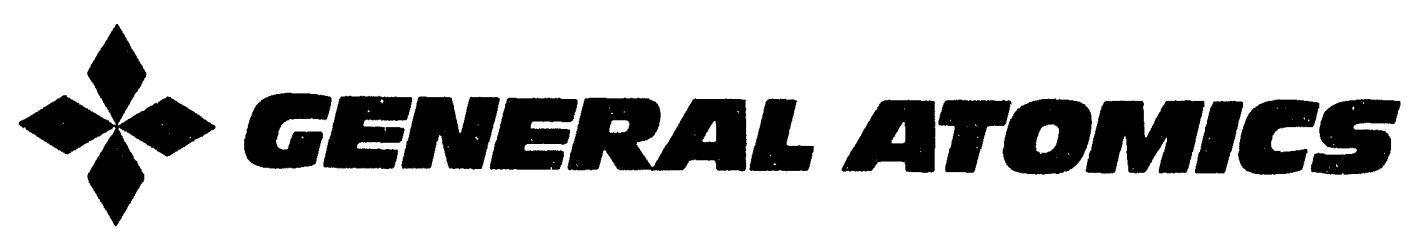




\title{
Experimental survey of the $\mathrm{L}-\mathrm{H}$ transition conditions in the DIII-D tokamak
}

\author{
T.N. Carlstrom, P. Gohil, J.G. Watkins, ${ }^{*}$ K.H. Burrell, S. Coda, ${ }^{\dagger}$ \\ E.J. DOYLE, R.J. Groebner, J. Kim, R.A. MOYER, C.L. RetTig \\ General Atomics, P.O. Box 85608, San Diego, California 92186-9784, U.S.A.
}

\begin{abstract}
We present the global analysis of a recent survey of the H-mode power threshold in DIII-D using $D^{\circ} \rightarrow D^{+}$NBI after boronization of the vacuum vessel. Single parameter scans of $B_{T}, I_{P}$, density, and plasma shape have been carried out on the DIII-D tokamak for neutral beam heated single-null and double-null diverted plasmas. In singlenull discharges, the power threshold is found to increase approximately linearly with $B_{T}$ and $n_{e}$ but remains independent of $I_{P}$. In double-null discharges, the power threshold is found to be approximately independent of both $B_{T}$ and $n_{e}$. Various shape parameters such as plasma-wall gaps had only a weak effect on the power threshold. Imbalancing the double null configuration resulted in a large increase in the threshold power.
\end{abstract}

\section{Introduction}

It is important to investigate the scaling of threshold conditions and the operating space of $\mathrm{H}$-mode in order to predict the confinement regime of future devices such as ITER. Measurements of the plasma conditions required to achieve H-mode also provide both inspiration and examination for theoretical models of the transition. Experiments in which one parameter is varied as others are held fixed are especially important in determining the parametric dependence and scaling of the $L-H$ transition. In this paper we present the global analysis of a recent survey of the $\mathrm{H}$-mode power threshold in DIII-D using $D^{\circ} \rightarrow D^{+}$NBI after boronization of the vacuum vessel. Detailed analysis of plasma edge conditions leading to a transition will be discussed in future work.

\section{Method}

The experiments were carried out in a freshly boronized vessel. Following standard operating procedure on DIII-D, helium glow discharge cleaning was used between shots to keep the vessel wall conditioned. A reference discharge was established with parameters set near the midrange of the operating space that can be scanned conveniently in DIII-D. The desired parameter was then scanned on a shot-to-shot basis with a stairstep power scan (300 ms/step) on each shot.

In order to lower the step size of neutral beam heating power to below the one source level of $2.4 \mathrm{MW}$ at $75 \mathrm{KV}$, the beam was modulated at $50 \mathrm{~Hz}(20 \mathrm{~ms}$ period) and the duty cycle adjusted to $25 \%, 50 \%, 75 \%$, and $100 \%$. This produced average powers of $0.6,1.2,1.8$, and 2.4 MW without changing the beam voltage and the corresponding beam deposition profile. Figure 1 shows waveforms of the modulated beam power, the average power, the line density, and the $\mathrm{D}_{\alpha}$ signal from the divertor region during an $\mathrm{L}$ to $\mathrm{H}$ transition. Since the beam slowing down time is of the order of 20-30 ms, and energy confinement times of 90-120 ms, the plasma was an effective integrator of the power, and no effect of the beam modulation was seen on

\footnotetext{
* Sandia National Laboratories.

$\uparrow$ Massachusetts Institute of Technology.

t University of California, Los Angeles.
} 


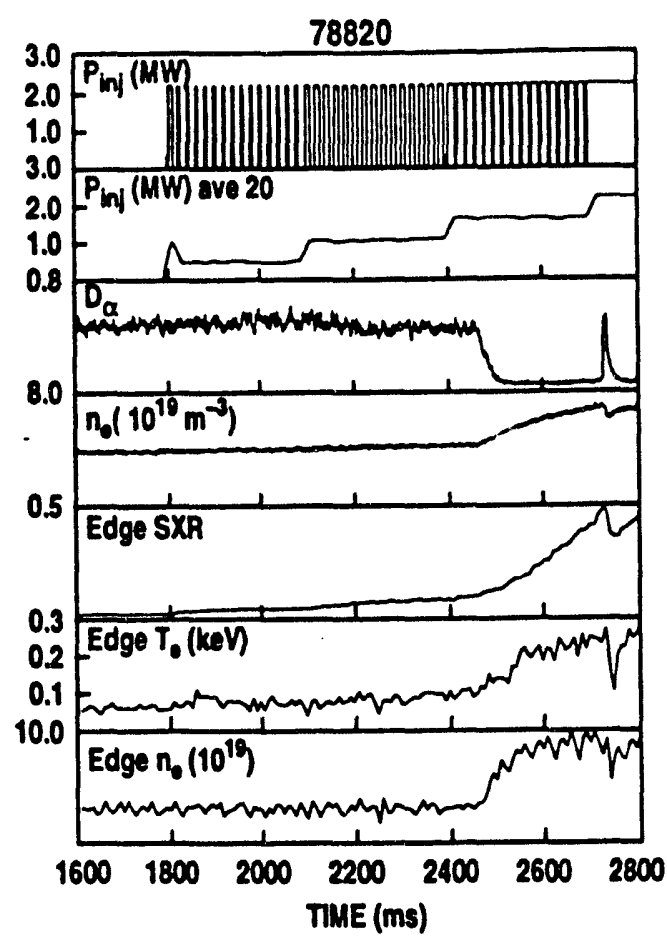

Fig. 1. Time evolution of a discharge showing the modulated NBI power, its average value over $20 \mathrm{~ms}$, and the steady condition of several edge plasma parameters in spite of the modulation. The L-H transition is at $2460 \mathrm{~ms}$. The edge $T_{e}$ and $n_{e}$ were measured with a multipulse Thomson effective measuring frequency of $120 \mathrm{~Hz}$. scattering system using six lasers with an

edge SXR, the $D_{\alpha}$ measurements, or the edge $T_{e}$ and $n_{e}$ measured by multipulse Thomson scattering.

Because threshold powers were often comparable to the Ohmic heating power, the power threshold includes both the Ohmic and absorbed neutral beam powers, Total Input Power $=P_{\text {nbi }}+P_{\text {ohmic }}$. $P_{\text {ohmic }}$ is calculated from smoothed $V_{\text {loop }} * I_{P}$. During the $\mathrm{L}$ phase, $V_{\text {loop }}$ is reasonably steady and comparisons between $V_{\text {loop }} * I_{P}$ and $O$ hmic power calculated from $Z_{\text {eft }}$ measurements and time dependent analysis of magnetics using EFIT and TAUFIT codes agree to an accuracy of $\pm 0.2 \mathrm{MW}$.

\section{Single-null results}

In single-null discharges, the power threshold is found to increase approximately linearly with $B_{T}$ and $n_{e}$ but varies only weakly with $I_{p}$. Figure $2(a)$ shows the equilibrium poloidal flux surfaces and various shape parameters for a lower single-null discharge. The $\nabla B$ drift direction is toward the $\mathrm{X}$-point. Figure 3 shows the individual scans of $B_{T}, n_{e}, I_{P}$, and outer gap. At the lowest toroidal field, Ohmic $\mathrm{H}$-mode was observed, and these data leverage the power threshold to vary approximately linearly with $B_{T}$. At $1.35 \mathrm{MA}$, Ohmic $\mathrm{H}$-modes were observed for $B_{T} \leq 1.6 \mathrm{~T}$ and input powers of approximately $1 \mathrm{MW}$. This point falls below the I-mode points shown in Fig. 3(a). Since Ohmic $\mathrm{H}$-modes in DIII-D are always triggered by a sawtooth crash, the modulation of power flow to the edge of the plasma by sawteeth may account for the lower threshold power. The density scan was severely limited at the low end by locked modes and at the high end by Marfes. Both these phenomena greatly increase the threshold power and those points were omitted from the scaling plot. Unfortunately, this left only a factor of two range in density and few data points. There remains, however, a definite increase in the threshold power with density. Dependence of the threshold power on $I_{P}$ was weak. The Ohmic power increased from $0.5 \mathrm{MW}$ at $1.0 \mathrm{MA}$ to $1.5 \mathrm{MW}$ at 2.0 MA with a nearly concomitant decrease in the NBI power. The edge safety factor $g_{95}$ changed from 6.2 at 1.0 MA to 3.1 at 2.0 MA. Decreasing the outer gap increased the threshold power weakly. Scans of X-point height, inside gap and a limited scan of the elongation, $\kappa$, showed no change in the power threshold over the range examined. The lack of any $\mathrm{X}$-point height dependence is inconsistent with our previous findings (Carlstrom et al., 1989) and is not yet understood. Table 1 summarizes the parameter scans, their range, and the effect of the threshold power.

\section{Double-null results}

In double-null discharges, the power threshold is found to be approximately independent of both $B_{T}$ and $n_{e}$. (The dependence on $I_{P}$ was not measured.) However, the threshold power is very sensitive to the balance between the two $\mathrm{X}$-points, measured by the parameter DrSEP, which is the distance between the separatrix surfaces associated with the two X-points, measured at the midplane. Figure 2(b) shows the flux surfaces for a balanced double-null, $\left(D r_{\text {SEP }}=0.0\right)$, and 
(a)

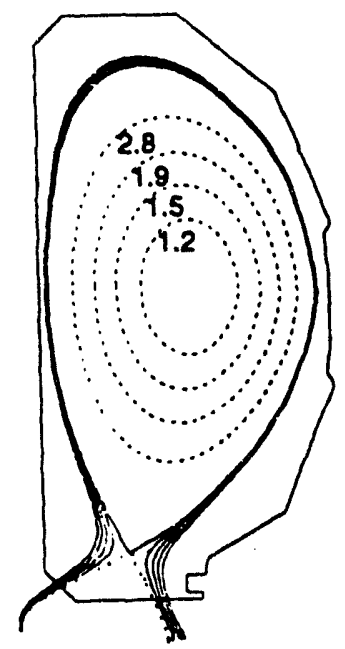

(c)

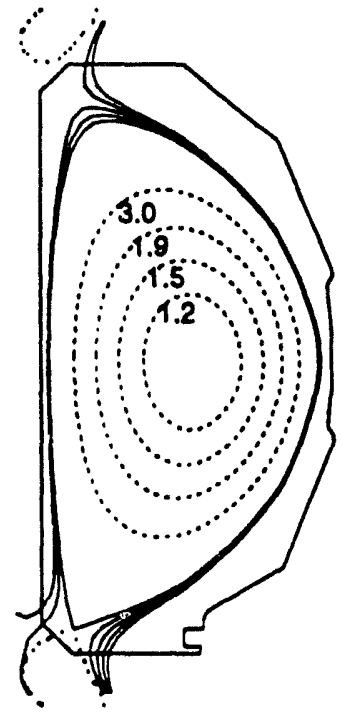

(b)

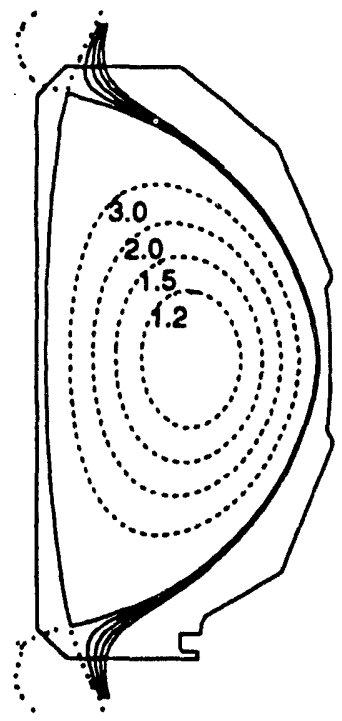

(d)

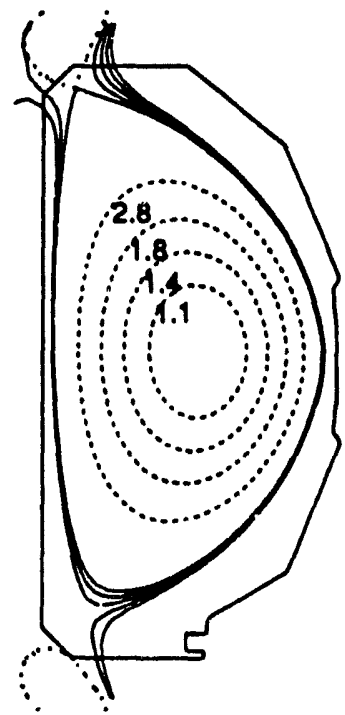

Fig. 2. Poloidal flux contours obtained from an equilibrium reconstruction code based on magnetic measurment are shown for four cases: (a) single-null discharge with $X$-point in direction of $\nabla \mathrm{B}$ drift, (b) balanced double-null discharge $\left(D r_{\text {SEP }}=0.0 \mathrm{~cm}\right)$, (c) imbalanced double-null discharge with lower $X$-point dominant $\left(D r_{\text {SEP }}=-0.6 \mathrm{~cm}\right)$, and (d) imbalanced double-null discharge with upper $X$-point dominant $\left(D r_{\mathrm{SEP}}=0.6 \mathrm{~cm}\right)$.

the midplane. Figure 2(b) shows the flux surfaces for a balanced doublenull, $\left(D r_{\text {SEP }}=0.0\right)$, and the imbalanced double-nulls are shown in Figs. 2(c) and (d). Figure 4 shows the double-null parameter scans of $B_{T}, n_{e}, D r_{\mathrm{SEP}}$ and inside gap. The threshold power does not change as the plasma is imbalanced toward the X-point in the $\nabla B$ drift direction. However, the power threshold increases from $4 \mathrm{MW}$ to over $14 \mathrm{MW}$ when the plasma is imbalanced toward the $\mathrm{X}$ point away from the $\nabla B$ drift direction. This scan showed the most dramatic effect on the power threshold in our survey. Table 2 summarizes the double-null parameter scans, their range, and the effect of the threshold power.

\section{Discussion and conclusions}

The use of deuterium beams and advances in wall conditioning techniques such as boronization and helium glow discharge cleaning before each plasma discharge has resulted in $40 \%-60 \%$ lower power thresholds than previously reported using hydrogen beams (Carlstrom et al., 1989). The threshold power in double-null discharges scales differently than single null discharges. The lowest power thresholds were found in single-null discharges at low field where the Ohmic heating power was sufficient to obtain H-mode. Ohmic H-modes have not been observed in the doublenull configuration. Figure 5 shows the threshold power normalized to the plasma surface area $\left\{S=4 \pi^{2} a R\left[\left(1+\kappa^{2}\right) /\right.\right.$ $\left.2]^{1 / 2}\right\}$ plotted against $n B$ for single and double-null discharges. This scaling relation and the proportionality constant for single-null discharges is consistent with the results from other devices (Ryter et al., 1993).

A comparison of the total radiated power in single-null discharges showed almost no change from our previous survey. Because of the low power thresholds in the current survey, the fraction of total radiated power has actually increased.

The threshold power scales with $B_{T}$ in the single-null configuration but is only weakly dependent on $B_{T}$ in the double null configuration. This suggests that the power threshold is probably not due to an intrinsic plasma parameter within the separatrix that may depend on the toroidal field such as the gyro radius, but rather that phenomena at the plasma edge or in the SOL plasma that depends on the field line geometry may be responsible. The lack of $B_{T}$ 

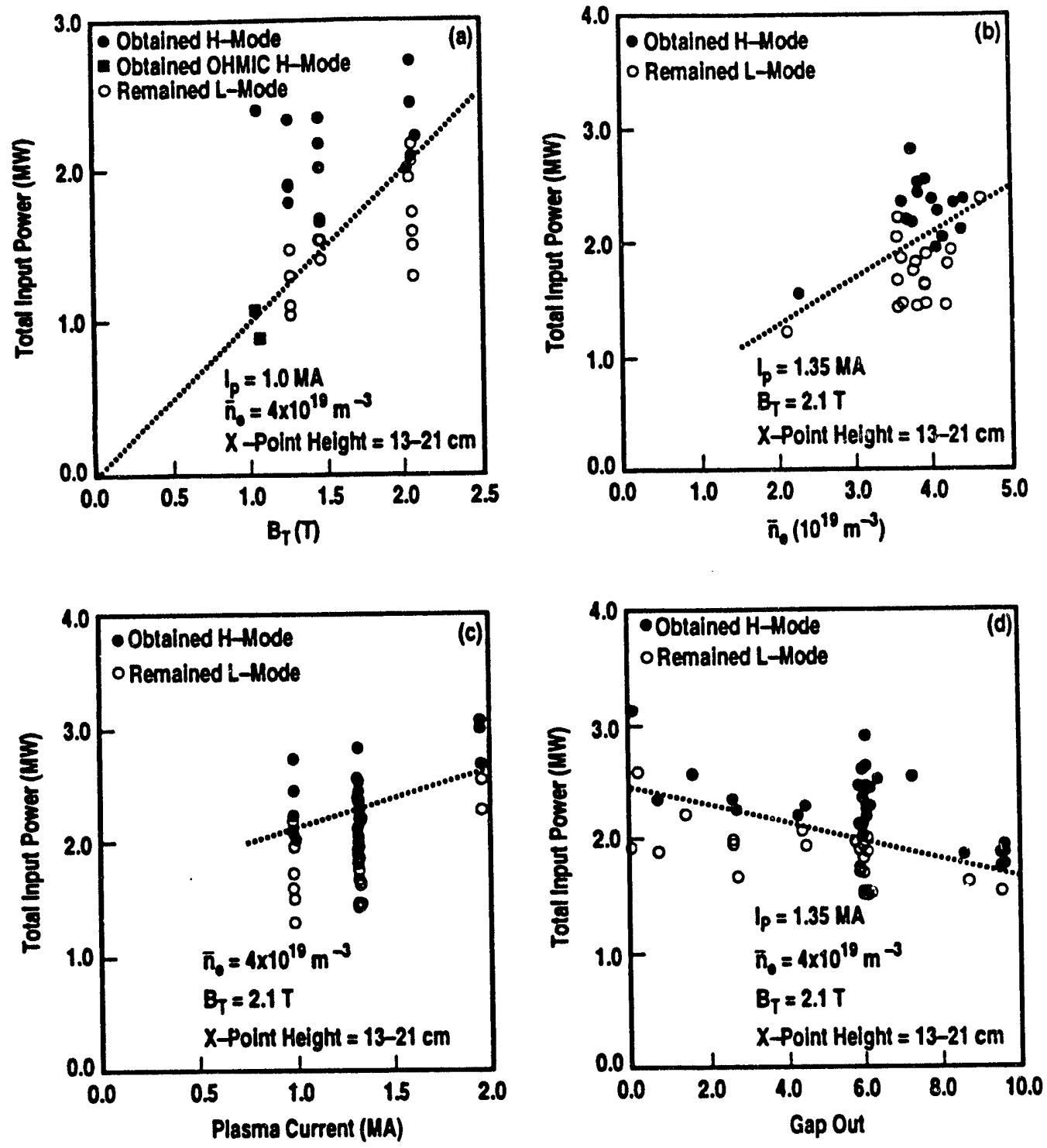

Fig. 3. Single-null threshold power scans of (a) $B_{T}$, (b) $\bar{n}_{e}$, (c) $I_{p}$ and (d) the outer gap.

Table 1

Single-null threshold power survey

\begin{tabular}{llll}
\hline & Parameter & \multicolumn{1}{c}{ Range } & Effect on Power Threshold \\
\hline 1. & $B_{T}$ & 1.0 to $2.1($ Telsa) & Increases roughly linearly \\
2. & $n_{e}$ & 2.0 to $5.0\left(10^{18} \mathrm{~m}^{-3}\right)$ & Increases \\
3. & $I_{p}$ & 1.0 to $2.0(\mathrm{MA})$ & Increases weakly \\
4. & X-point height & 0.0 to $25(\mathrm{~cm})$ & No dependence \\
5. & $\kappa$ & 1.78 to 2.04 & No dependence \\
6. & Inside Gap & 0.3 to $4.5(\mathrm{~cm})$ & No dependence \\
7. & Outside Gap & 0.0 to $10(\mathrm{~cm})$ & Weakly decreases \\
\hline
\end{tabular}



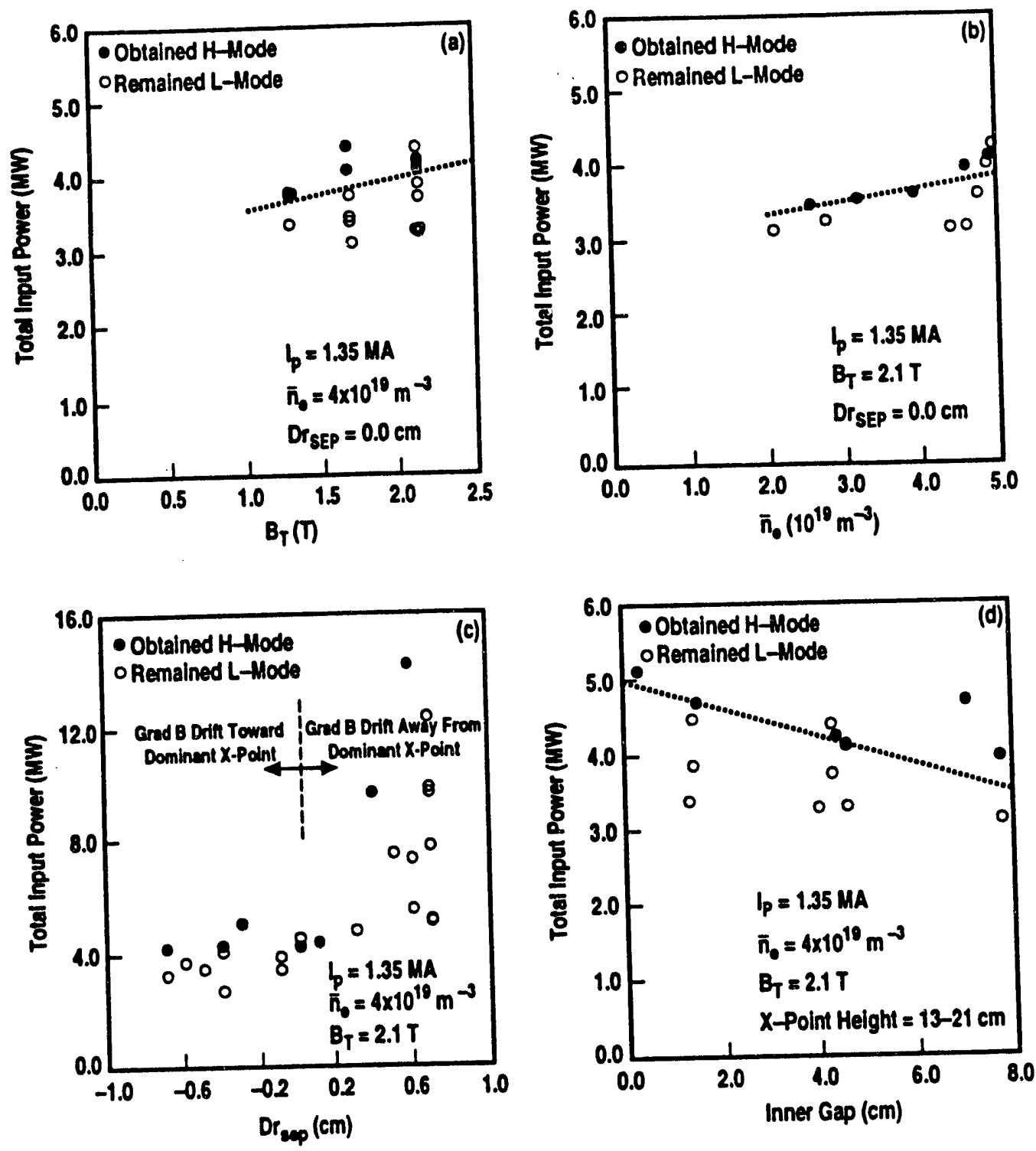

Fig. 4. Double-null threshold power scans of (a) $B_{T},(b) \bar{n}_{e},(c) D r_{S E P}$, and (d) the inner gap.

Table 2

Double-null threshold power survey

\begin{tabular}{llll}
\hline & Parameter & \multicolumn{1}{c}{ Range } & Effect on Power Threshold \\
\hline 1. & $B_{T}$ & 1.3 to $2.1($ Telsa $)$ & Weakly increases \\
2. & $n_{e}$ & 2.0 to $5.0\left(10^{10} \mathrm{~m}^{-3}\right)$ & Weakly increases \\
3. & DrSEP & -0.8 to $+0.8(\mathrm{~cm})$ & Strong dependence for DrSEP $>0$ \\
4. & Inside Gap & 1.0 to $8.0(\mathrm{~cm})$ & Weakly decrease
\end{tabular}

dependence in the double-null case may be consistent with earlier ASDEX results which did not see a $B_{T}$ dependence either (Wagner et al., 1989). ASDEX was a circular machine that may have always acted like a double-null (see $D r_{\text {SEP }}$ scan). Although the effect of the relationship 


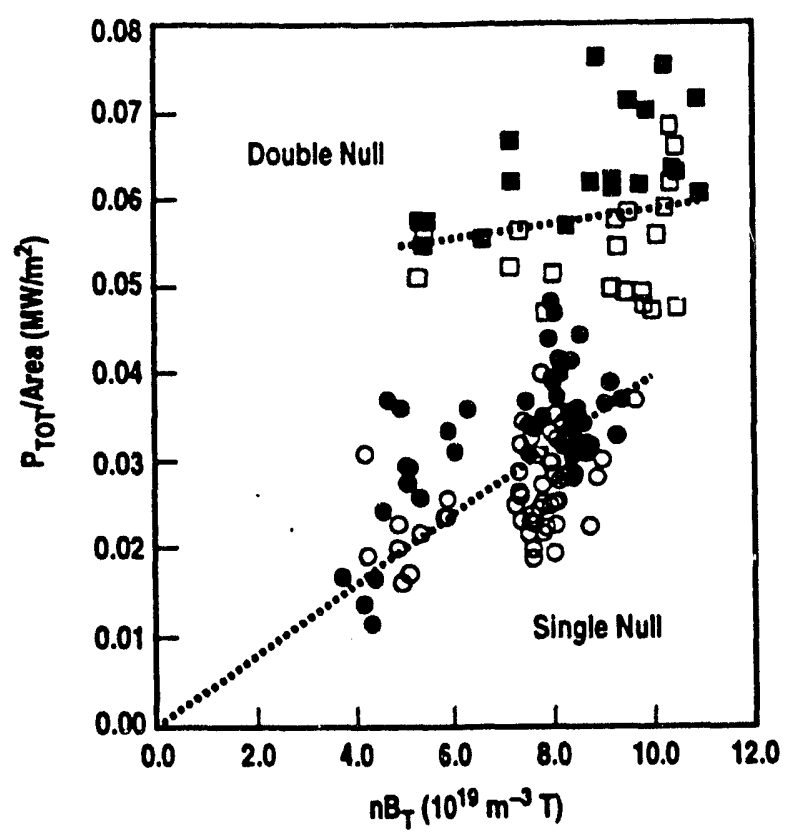

Fig. 5. Total input power normalized to the plasma surface area showing the $n B$ scaling for singlenull discharges and the lack of $n B$ scaling for double-null discharges. Open points remained L-mode, filled points obtained $\mathrm{H}$-mode. between the $\nabla \mathrm{B}$ drift direction and the $\mathrm{X}$ point location has been observed in many devices, the effect in this survey was very pronounced. For double-null discharges, the $\nabla B$ drifts and their relationship to the $\mathrm{X}$-point have a dominant role in determining the threshold power. The lack of dependence on the X-point height in single-null is inconsistent with our previous results and operating experience. Boronization may have changed effects of radiation in the divertor which in turn, changed the $\mathrm{X}$-point dependence.

It is interesting to use the present scalings to estimate the power thresholds for ITER. For single null, $P_{\text {th_SN }}(\mathrm{MW})$ $\sim 0.04 n\left(10^{20} \mathrm{~m}^{-3}\right) B(\mathrm{~T}) S\left(\mathrm{~m}^{2}\right)$. Using values from the ITER CDA (ITER, 1991) of $n=1, B=4.85, a=2.15, R=6$, and $\kappa=2$, we obtain $P_{\text {th_SN }}$ (ITER) $\sim 156(\mathrm{MW})$. For double null, $P_{t h \_N}(\mathrm{MW}) \sim 0.06 S\left(\mathrm{~m}^{2}\right)$ and $P_{t h \_D N}($ ITER $) \sim 48(\mathrm{MW})$. Having a single null threshold higher than a double null is inconsistent with with the results from many machines which find the lowest thresholds for

single null. This suggests that the $n B$ scaling of $P_{t h}$ for single null may saturate at higher $n B$ values. The ITER value for $n B$ is about 5 times the highest DIII-D value and $S$ is about 13 times greater. Accordingly, accurate size scaling of $P_{t h}$ as well as plasma parameter scaling is important for predicting $P_{t h}$ for ITER.

Although there is some consistency in single-null discharges for the threshold power to scale with $n B$, more work remains to be done before an understanding of the conditions leading to an $\mathrm{L}-\mathrm{H}$ transition is achieved.

\section{Acknowledgment}

This work was supported by the U.S. Department of Energy under Contract Nos. DE-AC0389ER-51114, DE-FG03-89ER51121, DE-AC02-78ET51013, and DE-AC03ั-76DP00789.

\section{References}

Carlstrom T N et al. 1989 Controlled Fusion and Plasma Physics Vol. 13B, p. 241. ITER Tokamak Device 1991 ITER Documentation Series, No. 25 (LAEA, Vienna) Ryter F et al. 1993 20th European Conf. on Controlled Fusion and Plasma Physics; Hutchinson I et al. 1993 35th Annual Meeting of the Division of Plasma Physics.

Wagner F 1989 Proceedings of 1989 IAEA Technical Committee Meeting on H-mode Physics, Plasma Phys. and Contr. Fusion. 

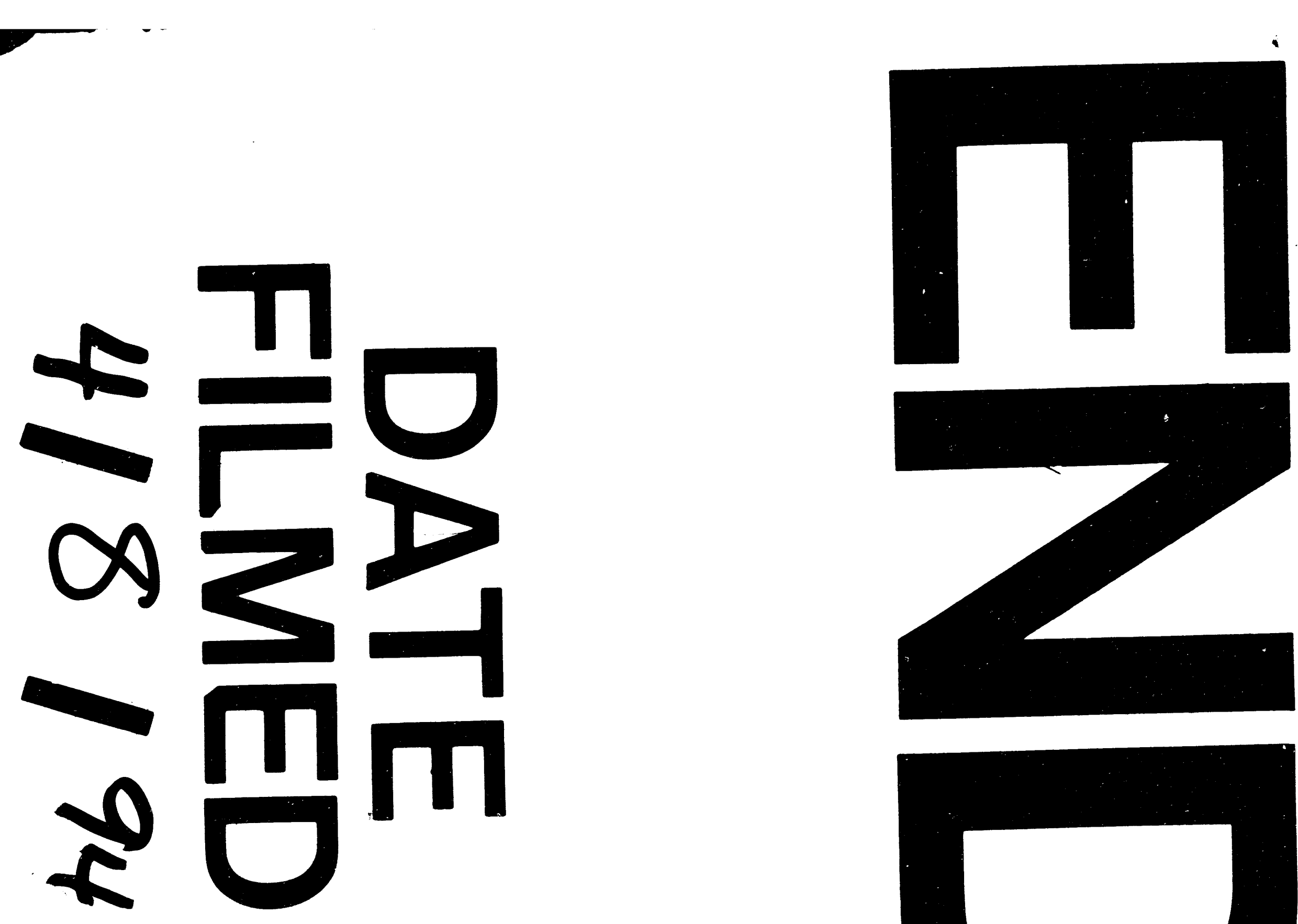

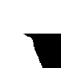


